\title{
A CENTRAL LIMIT PROPERTY UNDER A MODIFIED EHRENFEST URN DESIGN
}

\author{
YUNG-PIN CHEN, ${ }^{*}$ Lewis \& Clark College
}

\begin{abstract}
We consider a stochastic process in a modified Ehrenfest urn model. The modification prescribes there to be a minimum number of balls in each urn, and the process records the differences between treatment assignments under a sampling scheme implemented with this modified Ehrenfest urn model. In contrast to the result that the difference process forms a Markov chain and converges to a stationary distribution under the Ehrenfest urn model, the corresponding process under this modified Ehrenfest urn design satisfies the central limit property. We prove this asymptotic normality property using a central limit theorem for dependent random variables, renewal theory, and two Kolmogorov-type maximal inequalities.
\end{abstract}

Keywords: Ehrenfest urn model; Markov chain; renewal theory; central limit property; maximal inequality

2000 Mathematics Subject Classification: Primary 60F05

Secondary 60J10; 60K05

\section{Introduction}

In 1907, Paul and Tatiana Ehrenfest, two physicists, proposed an urn model that describes the diffusion of molecules through a membrane between two isolated bodies [8]. In their model, there are in total $2 w$ balls $(w \geq 1)$ distributed between two urns, which represent the two bodies. One ball is randomly chosen at a time. This ball is removed from its urn and placed in the other urn. These steps are repeated. If $W_{n}$ denotes the number of balls in one urn after $n$ steps, then $W_{n}$ forms a Markov chain on the state space $\{0,1,2, \ldots, 2 w\}$, with 0 and $2 w$ as its two completely reflecting barriers. The process $W_{n}$ is known as the Ehrenfest chain.

In the Ehrenfest urn model, the probability that one urn will lose a ball to the other is proportional to the number of balls in the first urn. Thus, there is a tendency toward balance in the process of ball transitions. Feller interpreted this transition tendency toward balance as diffusion with a central force [9, pp. 377-378]. Bingham [4] focused on the fluctuation theory that analyzes the stochastic behavior of the first passage time between the two extreme states under the Ehrenfest urn design. In [15] and [16], as sequels to [4], Palacios elegantly used the electric network approach to study the Ehrenfest urn. Besides playing an important role in statistical mechanics and its many applications in other, related fields, the Ehrenfest urn model is employed to assign treatments in clinical trials and is found to be quite favorable, in terms of randomness and balance properties, when compared with the sampling designs implemented with a biased coin [6]. The trial settings and the Ehrenfest urn design (EUD) can be described as follows.

Received 11 October 2005; revision received 9 January 2006.

* Postal address: Department of Mathematical Sciences, Lewis \& Clark College, Portland, OR 97219, USA.

Email address: ychen@1clark.edu 
1. Trial settings. Two treatments, say 1 and 2 , are assigned to sequentially arriving subjects in a trial. Suppose that each assignment is not necessarily adapted to the treatment responses. The clinician looks for a design that forces treatment assignments to be sufficiently balanced while simultaneously remaining sufficiently random.

\section{Ehrenfest urn design.}

(i) Each of the two urns, respectively labeled 1 and 2, initially contains $w$ balls $(w \geq 1)$.

(ii) One ball is chosen at random from the $2 w$ balls. The selection of a ball from urn $i$ corresponds to the assignment of treatment $i, i=1,2$.

(iii) The chosen ball is removed from its urn and is placed in the other urn.

(iv) The operations in (ii) and (iii) are repeated until the trial is terminated.

Let $W_{n, i}$ be the number of balls in urn $i, i=1,2$, after $n$ steps. The Ehrenfest urn design is then determined by the sequence of probability vectors $\left(W_{n, 1} / 2 w, W_{n, 2} / 2 w\right)$. Let $T_{n, i}$ be the number of times treatment $i$ is assigned after $n$ steps, and let $\Delta_{n}=T_{n, 1}-T_{n, 2}$ denote the net difference between treatment assignments after $n$ steps. It can be shown that

$$
\Delta_{n}=w-W_{n, 1}
$$

That is, under the EUD, the difference between treatment assignments after $n$ steps is determined by the difference between the initial number of balls, $w$, and the number of balls in either urn after $n$ steps. Note that $\Delta_{n}$ is a Markov chain on the state space $\{-w,-w+1, \ldots,-1,0,1, \ldots, w\}$ because $W_{n, 1}$ is an Ehrenfest chain.

The difference process, $\Delta_{n}$, provides a measure of how balanced a design is. It was shown in [6] that the EUD is more balanced than the biased coin design when both designs are held to have the same selection bias (defined in [5]). A major source of selection bias under the EUD comes from when an urn is empty and a deterministic treatment assignment has to be made. To avoid such deterministic treatment assignments, we modify the EUD as follows.

\section{Modified Ehrenfest urn design (MEUD).}

(i) Both urn 1 and urn 2 initially contain $w$ balls ( $w \geq 1)$. In addition, we specify an integer $v, 0<v<w$, to be the minimum number of balls each urn should at all times contain.

(ii) When $v<W_{n, 1}<2 w-v$, we follow the EUD.

(iii) When $W_{n, 1}=v$ (and, thus, $W_{n, 2}=2 w-v$ ),

(a) we set $\left(W_{n+1,1}, \Delta_{n+1}\right)=\left(v, \Delta_{n}+1\right)$ if a ball from urn 1 is selected;

(b) we set $\left(W_{n+1,1}, \Delta_{n+1}\right)=\left(v+1, \Delta_{n}-1\right)$ if a ball from urn 2 is selected.

When $W_{n, 1}=2 w-v$ (and, thus, $W_{n, 2}=v$ ), we perform similar operations.

(iv) The operations in (ii) and (iii) are repeated until the trial is terminated.

We will call this scheme of assigning treatments a modified Ehrenfest urn design with an initial number of balls $w$ and minimum number of balls $v$, and denote it by $\operatorname{MEUD}(w, v)$. 
Note that if, on the one hand, we set $v=0$, then $\operatorname{MEUD}(w, v=0)$ reduces to the EUD and $\left|\Delta_{n}\right|$ converges weakly to the distribution with the probability mass function

$$
p_{0}=\left(\begin{array}{c}
2 w \\
w
\end{array}\right)\left(\frac{1}{2}\right)^{2 w}, \quad p_{j}=2\left(\begin{array}{c}
2 w \\
j
\end{array}\right)\left(\frac{1}{2}\right)^{2 w}, \quad j=1,2, \ldots, w .
$$

If, on the other hand, we set $v=w$, then $\operatorname{MEUD}(w, v=w)$ becomes the repeated simple random sampling design, and $\Delta_{n}$ is the sum of independent and identically distributed random variables assuming the values 1 and -1 with equal probability. In this case, $\Delta_{n} / \sqrt{n}$ converges weakly to the standard normal distribution. The main goal of this paper is to show the central limit property of the net difference process, $\Delta_{n}$, under $\operatorname{MEUD}(w, v)$ with $0<v<w$.

Note further that $W_{n, 1}$, the number of balls in urn 1, forms a Markov chain on the state space $\{v, v+1, \ldots, 2 w-v\}$ with partially reflecting barriers $v$ and $2 w-v$. We will call $v+1$, $v+2, \ldots, 2 w-v-1$ the interior states. When $W_{n, 1}$ hits barrier $v$ (and, thus, $W_{n, 2}$ hits barrier $2 w-v$ ), it may stay there for a random number of steps. The simple equation stated in (1) no longer holds if $v>0$.

The paper is organized as follows. In Section 2, we will present a decomposition of $\Delta_{n}$ into three components. The major component is a random sum of stationary random variables. In Section 3, we use a central limit theorem for dependent summands to give a basic approximation of the major component of $\Delta_{n}$. Then we apply renewal theory to compute the asymptotic variance, in Section 4. In Section 5, two maximal inequalities are used to establish the central limit property of $\Delta_{n}$. We conclude with some remarks and future research, in Section 6.

\section{A decomposition of the difference process, $\Delta_{n}$}

To analyze $\Delta_{n}$ under $\operatorname{MEUD}(w, v)$, we need to look at two different events. The first event is when $W_{n, 1}$ stays at an interior state, i.e. when $v<W_{n, 1}<2 w-v$. In this case, as for the EUD, we find that $\Delta_{n}=w-W_{n, 1}$. The second event is when $W_{n, 1}$ hits either barrier $v$ or barrier $2 w-v$ (and may thus stay there for a random number of steps). When $W_{n, 1}$ hits barrier $v$ or $2 w-v$ from an interior state, the net difference $\Delta_{n}$ will increase or, respectively, decrease by 1 . This unit change is also accounted for by the quantity $w-W_{n, 1}$. The part that is not accounted for by $w-W_{n, 1}$ is the random number of steps for which $W_{n, 1}$ stays at the barrier. Let $W_{n}^{*}=\max \left(W_{n, 1}, W_{n, 2}\right)$, and define the following stopping times:

$$
\begin{aligned}
\tau_{0} & =0, & & \\
\tau_{k} & =\inf \left\{j>\tau_{k-1}: W_{j-1}^{*}<2 w-v, W_{j}^{*}=2 w-v\right\}, & & k \geq 1, \\
\eta_{k} & =\inf \left\{j>\tau_{k}: W_{j-1}^{*}=2 w-v, W_{j}^{*}<2 w-v\right\}, & & k \geq 1 .
\end{aligned}
$$

Of these, $\tau_{k}$ records the time at which $W_{n, 1}$ hits a barrier from an interior state for the $k$ th time and $\eta_{k}$ is the time at which $W_{n, 1}$ leaves that barrier after hitting it for the $k$ th time. The amount of time (measured as the number of steps) that $W_{n, 1}$ stays at a barrier after the $k$ th hit, excluding the step in which $W_{n, 1}$ hits that barrier, is

$$
G_{k}=\eta_{k}-\tau_{k}-1, \quad k \geq 1 .
$$

Note that $G_{k}, k \geq 1$, are independent and identically geometrically distributed random variables counting the number of steps stayed (i.e. failures) by $W_{n, 1}$ at a barrier before it leaves there (i.e. before the first success occurs). The probability that $W_{n, 1}$ departs from a barrier is $1-v / 2 w$. 
The quantity $G_{k}$ contributes positively or negatively to $\Delta_{n}$ according to whether $W_{n, 1}$ hits barrier $v$ or $2 w-v$. Now consider the process

$$
H_{k}=\frac{w-W_{\tau_{k}, 1}}{w-v}, \quad k \geq 1,
$$

which indicates whether a positive or a negative contribution is made to $\Delta_{n}$. It follows from the strong Markov property that the process $H_{k}$ is a Markov chain on the state space $\{1,-1\}$. Let

$$
\phi=\operatorname{Pr}\left\{H_{k+1}=1 \mid H_{k}=1\right\}
$$

be a one-step transition probability of $H_{k}$. It can be seen that $\phi$ is the probability that $W_{n, 1}$ hits barrier $v$ before barrier $2 w-v$, conditional on $W_{n, 1}$ starting at $v+1$. A routine calculation [10, pp. 29-31] for the birth-and-death chain $W_{n, 1}$ shows that

$$
\begin{aligned}
\phi & =\operatorname{Pr}\left\{H_{k+1}=1 \mid H_{k}=1\right\}=\operatorname{Pr}\left\{H_{k+1}=-1 \mid H_{k}=-1\right\} \\
& =\frac{\sum_{j=v+1}^{2 w-v-1}\left(\begin{array}{c}
2 w-1 \\
j
\end{array}\right)^{-1}}{\sum_{j=v}^{2 w-v-1}\left(\begin{array}{c}
2 w-1 \\
j
\end{array}\right)^{-1}}=1-\frac{\left(\begin{array}{c}
2 w-1 \\
v
\end{array}\right)^{-1}}{\sum_{j=v}^{2 w-v-1}\left(\begin{array}{c}
2 w-1 \\
j
\end{array}\right)^{-1}}
\end{aligned}
$$

and

$$
1-\phi=\operatorname{Pr}\left\{H_{k+1}=-1 \mid H_{k}=1\right\}=\operatorname{Pr}\left\{H_{k+1}=1 \mid H_{k}=-1\right\}=\frac{\left(\begin{array}{c}
2 w-1 \\
v
\end{array}\right)^{-1}}{\sum_{j=v}^{2 w-v-1}\left(\begin{array}{c}
2 w-1 \\
j
\end{array}\right)^{-1}} .
$$

Note that $\frac{1}{2} \leq \phi<1$ and, hence, $0 \leq 2 \phi-1<1$. We will use these inequalities later. Also, because both urns start with the same number of balls and the two barriers, $v$ and $2 w-v$, are equally likely to be visited by $W_{n, 1}, H_{k}$ has the initial distribution

$$
\operatorname{Pr}\left\{H_{1}=-1\right\}=\operatorname{Pr}\left\{H_{1}=1\right\}=\frac{1}{2} .
$$

This initial distribution is also the stationary distribution of $H_{k}$, so $H_{n}$ is a stationary process.

Finally, care must be taken in formulating the last time $W_{n, 1}$ hits a barrier after $n$ steps. Let

$$
N_{n}=\sup \left\{k: \tau_{k} \leq n\right\}
$$

count the number of times $W_{n, 1}$ hits a barrier after $n$ steps. Two different scenarios can occur between time $\tau_{N_{n}}$ and time $n$. One scenario is that $W_{n, 1}$ may stay at a barrier the whole time. In this case we have $\tau_{N_{n}} \leq n<\eta_{N_{n}}$, and the quantity contributed to $\Delta_{n}$ is $H_{N_{n}}\left(n-\tau_{N_{n}}\right)$. The other scenario is that $W_{n, 1}$ may have left the barrier it most recently visited. This means that $\tau_{N_{n}}<\eta_{N_{n}} \leq n$, and the quantity contributed to $\Delta_{n}$ is $H_{N_{n}}\left(\eta_{N_{n}}-\tau_{N_{n}}-1\right)=H_{N_{n}} G_{N_{n}}$. (Once $W_{n, 1}$ is at an interior state, $w-W_{n, 1}$ will again account for the difference.) The absolute change in $\Delta_{n}$ is thus $n-\tau_{N_{n}}$ or $G_{N_{n}}$, whichever is smaller.

By combining the above results, we can explicitly decompose $\Delta_{n}$ as follows:

$$
\Delta_{n}=\left(w-W_{n, 1}\right)+\sum_{k=1}^{N_{n}-1} H_{k} G_{k}+H_{N_{n}} \min \left(n-\tau_{N_{n}}, G_{N_{n}}\right) .
$$

Under the EUD, whenever $W_{n, 1}$ hits a barrier it reflects back to the nearest interior state with probability 1 . Hence, $G_{k}$ is 0 for all $k$ and (8) reduces to (1). 
Since we are interested in the asymptotic behavior of $\Delta_{n}$ under $\operatorname{MEUD}(w, v)$, we ask whether there exist constants $\mu_{n}$ and $\sigma_{n}$ such that $\left(\Delta_{n}-\mu_{n}\right) / \sigma_{n}$ converges weakly. The first term, $w-W_{n, 1}$, on the right-hand side of (8) is a finite Markov chain, so $n^{-r}\left(w-W_{n, 1}\right)$ converges weakly (in fact almost surely) to 0 for any $r>0$. The magnitude of the last term on the right-hand side of (8) is no larger than a truncated geometric random variable and, hence, also converges weakly to 0 if divided by $n^{-r}$, for any $r>0$. Regarding the middle term, it can be shown that the counting process, $N_{n}$, in (7), which is a delayed renewal process, tends to $\infty$ almost surely as $n \rightarrow \infty$. Therefore, when $n$ is large, $\Delta_{n}$ is essentially dominated by the series $\sum_{k=1}^{N_{n}-1} H_{k} G_{k}$. We will now show that the summands $H_{k} G_{k}$ form a strictly stationary sequence.

Theorem 1. Let $G_{k}$ and $H_{k}$ be respectively defined as in (4) and (5). Then $\left\{H_{k} G_{k}\right\}$ forms a strictly stationary sequence with $\mathrm{E}\left(H_{k} G_{k}\right)=0$ for all $k \geq 1$.

Proof. We have noted that $G_{k}, k \geq 1$, are independent and identically geometrically distributed random variables. For the process $H_{k}$, we have shown it to be a Markov chain on $\{-1,1\}$ with initial stationary distribution $\operatorname{Pr}\left\{H_{1}=-1\right\}=\operatorname{Pr}\left\{H_{1}=1\right\}=\frac{1}{2}$. Hence, $H_{k}$ is strictly stationary. In addition, the number of steps for which $W_{n, 1}$ stays at a barrier is independent of which barrier it visits, so $G_{k}$ and $H_{k}$ are independent for all $k \geq 1$. This proves the strict stationarity of $H_{k} G_{k}$ and shows that $\mathrm{E}\left(H_{k} G_{k}\right)=\mathrm{E}\left(H_{k}\right) \mathrm{E}\left(G_{k}\right)=\mathrm{E}\left(H_{1}\right) \mathrm{E}\left(G_{k}\right)=0$ for all $k \geq 1$.

\section{A central limit property of $S_{n}=\sum_{k=1}^{n} H_{k} G_{k}$}

Before tackling the series $\sum_{k=1}^{N_{n}-1} H_{k} G_{k}$, we will first consider $S_{n}=\sum_{k=1}^{n} H_{k} G_{k}$, a sum of a fixed number of stationary terms. Both [11] and [12] are good references for the central limit theorems for dependent random variables. We will show that $S_{n}$ satisfies the central limit property, using the results of [18]. Specifically, we use Theorem 4.1 and Corollary 4.1.1 of [18] because we can identify the asymptotic variance in the process of establishing the central limit property.

Lemma 1. Let $\left\{X_{j}\right\}$ be a sequence of random variables, and let $S_{a, n}=\sum_{j=a+1}^{a+n} X_{j}$. Let $\mathcal{F}^{b}=\sigma\left(X_{a}, X_{a+1}, \ldots, X_{b}\right)$ be the $\sigma$-field generated by the random variables $X_{a}, X_{a+1}$, $\ldots, X_{b}$, where $-\infty \leq a \leq b \leq \infty$. If $\left\{X_{j}\right\}$ satisfies the following conditions then it has the central limit property.

(A1) $\mathrm{E}\left(X_{j}\right)=0$ for all $j$.

(A2) As $n \rightarrow \infty, \operatorname{var}\left(S_{a, n}\right) \sim n A^{2}$ uniformly in a, where $A>0$.

(A3) There are constants $\delta>0$ and $M, 0<M<\infty$, such that $\mathrm{E}\left(\left|X_{j}\right|^{2+\delta}\right) \leq M$ for all $j$.

(D1) $\mathrm{E}\left(\left|\mathrm{E}\left(n^{-1 / 2} S_{a, n} \mid \mathcal{F}_{-\infty}^{a}\right)\right|\right) \leq O\left(n^{-\theta_{1}}\right)$ uniformly in a for some $\theta_{1}>0$.

(D2) $\mathrm{E}\left(\left|\mathrm{E}\left(n^{-1} S_{a, n}^{2} \mid \mathcal{F}_{-\infty}^{a}\right)-\mathrm{E}\left(n^{-1} S_{a, n}^{2}\right)\right|\right) \leq O\left(n^{-\theta_{2}}\right)$ uniformly in a for some $\theta_{2}>0$.

Furthermore, $S_{0, n} /(\sqrt{n} A)$ converges weakly to the standard normal distribution.

Serfling [18] referred to conditions (A1), (A2), and (A3) as the basic assumptions and conditions (D1) and (D2) as the dependence restrictions, which require the mean deviations of the first and second conditional moments given the history $\mathcal{F}_{-\infty}^{a}$ up to time $a$ to converge to 0 
uniformly in $a$ as $n \rightarrow \infty$. We will verify that the sequence $\left\{H_{j} G_{j}\right\}$ satisfies each of the five conditions of this lemma.

Condition (A1) has been shown to hold in Theorem 1. To check condition (A2), it suffices to show that $\operatorname{var}\left(\sum_{j=1}^{n} H_{j} G_{j}\right) \sim n A^{2}$ because of the stationarity of $H_{j} G_{j}$. It can be shown that $H_{j}$ has the $n$th order transition probabilities

$$
\begin{gathered}
\operatorname{Pr}\left\{H_{n+1}=1 \mid H_{1}=1\right\}=\operatorname{Pr}\left\{H_{n+1}=-1 \mid H_{1}=-1\right\}=\frac{1}{2}+\frac{1}{2}(2 \phi-1)^{n}, \\
\operatorname{Pr}\left\{H_{n+1}=1 \mid H_{1}=-1\right\}=\operatorname{Pr}\left\{H_{n+1}=-1 \mid H_{1}=1\right\}=\frac{1}{2}-\frac{1}{2}(2 \phi-1)^{n},
\end{gathered}
$$

and the covariance $\operatorname{cov}\left(H_{i}, H_{j}\right)=\mathrm{E}\left(H_{i} H_{j}\right)=(2 \phi-1)^{j-i}, i<j$. Thus, we have

$$
\begin{aligned}
\operatorname{var}\left(\sum_{j=1}^{n} H_{j} G_{j}\right)= & \sum_{j=1}^{n} \operatorname{var}\left(H_{j} G_{j}\right)+2 \sum_{1 \leq i<j \leq n} \operatorname{cov}\left(H_{i} G_{i}, H_{j} G_{j}\right) \\
= & n \mathrm{E}\left(H_{1}^{2} G_{1}^{2}\right)+2 \sum_{1 \leq i<j \leq n} \sum_{j} \mathrm{E}\left(H_{i} G_{i} H_{j} G_{j}\right) \\
= & n\left[\operatorname{var}\left(G_{1}\right)+\mathrm{E}\left(G_{1}\right)^{2}\right]+2 \mathrm{E}\left(G_{1}\right)^{2} \sum_{j=2}^{n} \sum_{i=1}^{j-1}(2 \phi-1)^{j-i} \\
= & n\left[\frac{2 v w}{(2 w-v)^{2}}+\left(\frac{v}{2 w-v}\right)^{2}\right] \\
& +2\left(\frac{v}{2 w-v}\right)^{2}\left[(n-1) \frac{2 \phi-1}{2-2 \phi}-\frac{(2 \phi-1)^{2}}{(2-2 \phi)^{2}}+\frac{(2 \phi-1)^{n+1}}{(2-2 \phi)^{2}}\right] \\
\sim & n A(\phi, w, v)^{2},
\end{aligned}
$$

where

$$
A(\phi, w, v)^{2}=\frac{2 v w+v^{2}+(2 \phi-1) v^{2} /(1-\phi)}{(2 w-v)^{2}}=\frac{2 v w+\phi v^{2} /(1-\phi)}{(2 w-v)^{2}} .
$$

Note that the quantity $A(\phi, w, v)^{2}$ is positive unless $v=0$. Condition (A2) is thus satisfied.

Condition (A3) trivially holds because $\left|H_{j}\right|=1$ for all $j$ and $\left|H_{j} G_{j}\right|$ has the same distribution as $G_{1}$, which has moments of all orders.

Because $H_{j} G_{j}$ is Markovian and initially stays stationary at epoch $j=1$, it is enough to show that conditions (D1) and (D2) hold for $a=1$. We thus consider $\mathcal{F}_{-\infty}^{a}=\mathscr{F}_{-\infty}^{1}=\sigma\left(H_{1}, G_{1}\right)$ and let $S_{1, n}=\sum_{j=2}^{n+1} H_{j} G_{j}$. Because $G_{j}$ is a sequence of independent and identically geometrically distributed random variables independent of $H_{j}$, we have

$$
\mathrm{E}\left(H_{j} G_{j} \mid \sigma\left(H_{1}, G_{1}\right)\right)=\mathrm{E}\left(G_{j}\right) \mathrm{E}\left(H_{j} \mid H_{1}\right)=\frac{v}{2 w-v} H_{1}(2 \phi-1)^{j-1}, \quad j \geq 2 .
$$

Hence,

$$
\begin{aligned}
\left|\mathrm{E}\left(n^{-1 / 2} S_{1, n} \mid \mathcal{F}_{-\infty}^{1}\right)\right| & =\left|\mathrm{E}\left(n^{-1 / 2} \sum_{j=2}^{n+1} H_{j} G_{j} \mid H_{1}\right)\right| \\
& =n^{-1 / 2} \frac{v}{2 w-v}\left|H_{1}\right| \sum_{j=2}^{n+1}(2 \phi-1)^{j-1} .
\end{aligned}
$$


Because $0 \leq 2 \phi-1<1$, this verifies that condition (D1) holds with $\theta_{1}=\frac{1}{2}$. To verify that condition (D2) holds, it can be shown that $\mathrm{E}\left(H_{i} H_{j} \mid H_{1}\right)=(2 \phi-1)^{j-i}$ for all $i$ and $j, 1<i<j$, and that

$$
\mathrm{E}\left(S_{1, n}^{2} \mid \mathcal{F}_{\infty}^{1}\right)=\mathrm{E}\left(S_{1, n}^{2}\right)=\mathrm{E}\left(\left(\sum_{j=2}^{n+1} H_{j} G_{j}\right)^{2}\right)=A(\phi, w, v)^{2},
$$

the quantity given in (10). Clearly, condition (D2) holds.

Having verified that all the conditions of Lemma 1 hold, we deduce the following asymptotic normality of $S_{n}=\sum_{j=1}^{n} H_{j} G_{j}$.

Theorem 2. Let $G_{k}$ and $H_{k}$ be respectively defined as in (4) and (5) under the MEUD. The partial sum $S_{n}=\sum_{j=1}^{n} H_{j} G_{j}$ then has the central limit property. Moreover,

$$
\mathcal{L}\left(\frac{S_{n}}{\sqrt{n} A(\phi, w, v)}\right) \rightarrow \mathrm{N}(0,1), \quad \text { where } A(\phi, w, v)^{2}=\frac{2 v w+\phi v^{2} /(1-\phi)}{(2 w-v)^{2}},
$$

and $\phi$ is the transition probability of the chain $H_{n}$ given in (6).

\section{The limit of $N_{n} / n$}

Now we examine the process defined in (7), $N_{n}$, which is a delayed renewal process counting the number of times $W_{n, 1}$ hits a barrier after $n$ steps. It follows from renewal theory that $N_{n} / n$ converges to the reciprocal, $\theta$, of the mean interarrival time of the renewal process $N_{n}$ almost surely, as $n \rightarrow \infty$. We will compute $\theta$ in this section.

The interarrival time of the renewal process $N_{n}$ consists of two parts. The first part is the random number of steps for which $W_{n, 1}$ stays at barrier $v$ or barrier $2 w-v$, plus the one jump to the nearest interior state, $v+1$ or $2 w-v-1$. Let us denote this random number of steps by the random variable $X$. This in fact has the same distribution as $\tau_{k}-\eta_{k}$, where $\tau_{k}$ and $\eta_{k}$ are as defined in (2) and (3), respectively, and

$$
\mathrm{E}(X)=\frac{2 w}{2 w-v}
$$

The second part of $N_{n}$ is the random number of steps taken by $W_{n, 1}$ to return to a barrier since its last departure from one. It is the same as the number of steps $W_{n, 1}$ will take to hit either barrier when $W_{n, 1}$ starts at state $v+1$ (or $2 w-v-1$ ). We know that $W_{n, 1}$ is a birth-and-death chain on the set $\{v, v+1, \ldots, 2 w-v\}$. Let $p_{x}$ and $q_{x}$ respectively be the birth and death probabilities of $W_{n, 1}$, and let $\xi(x)$ be the mean time at which $W_{n, 1}$ will hit either barrier when starting at state $x \in\{v, v+1, \ldots, 2 w-v\}$ with the boundary conditions $\xi(v)=\xi(2 w-v)=0$. For $x \in\{v+1, \ldots, 2 w-v-1\}$, we have

$$
\begin{aligned}
\xi(x) & =q_{x}[\xi(x-1)+1]+p_{x}[\xi(x+1)+1] \\
& =q_{x} \xi(x-1)+p_{x} \xi(x+1)+1,
\end{aligned}
$$

which further implies that

$$
\xi(x)-\xi(x+1)=\frac{q_{x}}{p_{x}}[\xi(x-1)-\xi(x)]+\frac{1}{p_{x}} .
$$


We can inductively show that, for $x \in\{v+1, \ldots, 2 w-v-1\}$,

$$
\begin{aligned}
\xi(x)-\xi(x+1)= & \prod_{l=v+1}^{x} \frac{q_{l}}{p_{l}}[\xi(v)-\xi(v+1)] \\
& +\frac{q_{x} q_{x-1} \cdots q_{v+2}}{p_{x} p_{x-1} \cdots p_{v+1}}+\cdots+\frac{q_{x} q_{x-1}}{p_{x} p_{x-1} p_{x-2}}+\frac{q_{x}}{p_{x} p_{x-1}}+\frac{1}{p_{x}} \\
= & -\prod_{l=v+1}^{x} \frac{q_{l}}{p_{l}} \xi(v+1)+\sum_{l=v+2}^{x} \frac{1}{p_{l-1}} \prod_{j=l}^{x} \frac{q_{j}}{p_{j}}+\frac{1}{p_{x}} .
\end{aligned}
$$

By summing the above equations over $x=v+1, \ldots, 2 w-v-1$ and using the initial condition that $\xi(2 w-v)=0$, we obtain

$$
\xi(v+1)=-\xi(v+1) \sum_{x=v+1}^{2 w-v-1} \prod_{l=v+1}^{x} \frac{q_{l}}{p_{l}}+\sum_{x=v+1}^{2 w-v-1}\left[\frac{1}{p_{x}}+\sum_{l=v+2}^{x} \frac{1}{p_{l-1}} \prod_{j=l}^{x} \frac{q_{j}}{p_{j}}\right] .
$$

Hence,

$$
\xi(v+1)=\left(\sum_{x=v+1}^{2 w-v-1} \frac{1}{p_{x}}+\sum_{x=v+2}^{2 w-v-1} \sum_{l=v+2}^{x} \frac{1}{p_{l-1}} \prod_{j=l}^{x} \frac{q_{j}}{p_{j}}\right)\left(1+\sum_{x=v+1}^{2 w-v-1} \prod_{l=v+1}^{x} \frac{q_{l}}{p_{l}}\right)^{-1} .
$$

By making the substitutions $p_{x}=(2 w-x) / 2 w$ and $q_{x}=x / 2 w$, for $x \in\{v+1, \ldots, 2 w-v-1\}$, and letting

$$
\begin{aligned}
\gamma_{x}=\prod_{l=v+1}^{x} \frac{q_{l}}{p_{l}} & =\frac{(v+1)(v+2) \cdots x}{(2 w-v-1)(2 w-v-2) \cdots(2 w-x)} \\
& =\frac{x !(2 w-x-1) !}{v !(2 w-v-1) !}=\frac{\left(\begin{array}{c}
2 w-1 \\
v
\end{array}\right)}{\left(\begin{array}{c}
2 w-1 \\
x
\end{array}\right)}
\end{aligned}
$$

we conclude the following result.

Theorem 3. Under the MEUD the renewal process $N_{n}$, defined in (7), satisfies

$$
\frac{N_{n}}{n} \rightarrow \theta \equiv \theta(w, v) \quad \text { almost surely as } n \rightarrow \infty,
$$

where

$$
\begin{aligned}
& \theta^{-1}=\frac{2 w}{2 w-v}+\left(\sum_{x=v+1}^{2 w-v-1} \frac{2 w}{2 w-x}+\sum_{x=v+2}^{2 w-v-1} \sum_{l=v+2}^{x} \frac{2 w}{2 w-l+1} \frac{\gamma_{x}}{\gamma_{l-1}}\right)\left(1+\sum_{x=v+1}^{2 w-v-1} \gamma_{x}\right)^{-1} \\
& \text { with } \gamma_{x}=\left(\begin{array}{c}
2 w-1 \\
v
\end{array}\right) /\left(\begin{array}{c}
2 w-1 \\
x
\end{array}\right) .
\end{aligned}
$$

\section{A central limit property for $S_{N_{n}}$}

In this section, we will establish the central limit property for $S_{N_{n}}$ :

$$
\mathcal{L}\left(\frac{S_{N_{n}}}{A \sqrt{n \theta}}\right) \rightarrow \mathrm{N}(0,1),
$$

where $A$ and $\theta$ are respectively obtained from Theorem 2 and Theorem 3 . 
First, note that if $\lfloor x\rfloor$ denotes the largest integer not exceeding $x$, then $n \theta /\lfloor n \theta\rfloor \rightarrow 1$ as $n \rightarrow \infty$. Thus, it is enough to show that $S_{N_{n}} /(A \sqrt{\lfloor n \theta\rfloor})$ converges weakly to $\mathrm{N}(0,1)$. Second, note that

$$
\frac{S_{N_{n}}}{A \sqrt{\lfloor n \theta\rfloor}}=\frac{S_{\lfloor n \theta\rfloor}}{A \sqrt{\lfloor n \theta\rfloor}}+\frac{S_{N_{n}}-S_{\lfloor n \theta\rfloor}}{A \sqrt{\lfloor n \theta\rfloor}}
$$

and that, from Theorem $2, S_{\lfloor n \theta\rfloor} /(A \sqrt{\lfloor n \theta\rfloor})$ converges weakly to $\mathrm{N}(0,1)$. It thus suffices to show that $\left(S_{N_{n}}-S_{\lfloor n \theta\rfloor}\right) / \sqrt{\lfloor n \theta\rfloor}$ converges to 0 in probability as $n \rightarrow \infty$. Now let $\varepsilon>0$ be chosen arbitrarily and consider the following partition:

$$
\begin{aligned}
\left\{\left|S_{N_{n}}-S_{\lfloor n \theta\rfloor}\right| \geq \varepsilon \sqrt{\lfloor n \theta\rfloor}\right\}= & \left(\left\{\left|S_{N_{n}}-S_{\lfloor n \theta\rfloor}\right| \geq \varepsilon \sqrt{\lfloor n \theta\rfloor}\right\} \cap\left\{\left|N_{n}-\lfloor n \theta\rfloor\right| \geq \varepsilon^{3}\lfloor n \theta\rfloor\right\}\right) \\
& \cup\left(\left\{\left|S_{N_{n}}-S_{\lfloor n \theta\rfloor}\right| \geq \varepsilon \sqrt{\lfloor n \theta\rfloor}\right\} \cap\left\{\left|N_{n}-\lfloor n \theta\rfloor\right|<\varepsilon^{3}\lfloor n \theta\rfloor\right\}\right) .
\end{aligned}
$$

The occurrence of the event $\left\{\left|S_{N_{n}}-S_{\lfloor n \theta\rfloor}\right| \geq \varepsilon \sqrt{\lfloor n \theta\rfloor}\right\}$ with $\left|N_{n}-\lfloor n \theta\rfloor\right|<\varepsilon^{3}\lfloor n \theta\rfloor$ implies that

$$
\max _{|k-\lfloor n \theta\rfloor| \leq \varepsilon^{3}\lfloor n \theta\rfloor}\left|S_{k}-S_{\lfloor n \theta\rfloor}\right| \geq \varepsilon \sqrt{\lfloor n \theta\rfloor} .
$$

Thus, we have

$$
\begin{aligned}
& \operatorname{Pr}\left\{\left|S_{N_{n}}-S_{\lfloor n \theta\rfloor}\right| \geq \varepsilon \sqrt{\lfloor n \theta\rfloor}\right\} \\
& \quad \leq \operatorname{Pr}\left\{\left|N_{n}-\lfloor n \theta\rfloor\right| \geq \varepsilon^{3}\lfloor n \theta\rfloor\right\}+\operatorname{Pr}\left\{\max _{|k-\lfloor n \theta\rfloor| \leq \varepsilon^{3}\lfloor n \theta\rfloor}\left|S_{k}-S_{\lfloor n \theta\rfloor}\right| \geq \varepsilon \sqrt{\lfloor n \theta\rfloor}\right\} .
\end{aligned}
$$

The first term on the right-hand side of (11) tends to 0 as $n \rightarrow \infty$, because of the almost-sure convergence of $N_{n} / n$ to $\theta$. The main task here is to control the second term using a maximal inequality. This scheme for proving the central limit theorem of a random number of summands is outlined in an exercise in [2, p. 369]. We also need two results due to Billingsley to accomplish the main task.

Lemma 2. ([1, pp. 87-88], [3, pp. 105-106].) Suppose that $Y_{n}$ is a sequence of random variables. Let $S_{0}=0$ and let $S_{k}=Y_{1}+\cdots+Y_{k}, k \geq 1$. Also let $M_{n}=\max \left(\left|S_{1}\right|, \ldots,\left|S_{n}\right|\right)$, $m_{i j k}=\min \left(\left|S_{j}-S_{i}\right|,\left|S_{k}-S_{j}\right|\right)$, and $L_{n}=\max _{0 \leq i \leq j \leq k \leq n} m_{i j k}$. Then $M_{n} \leq 3 L_{n}+$ $\max _{k \leq n}\left|Y_{k}\right|$. Hence, for $\lambda>0$,

$$
\operatorname{Pr}\left\{M_{n} \geq 4 \lambda\right\} \leq \operatorname{Pr}\left\{L_{n} \geq \lambda\right\}+\operatorname{Pr}\left\{\max _{k \leq n}\left|Y_{k}\right| \geq \lambda\right\} .
$$

The above lemma states that a bound for $\operatorname{Pr}\left\{M_{n} \geq 4 \lambda\right\}$ can be obtained through bounds on both $\operatorname{Pr}\left\{L_{n} \geq \lambda\right\}$ and $\operatorname{Pr}\left\{\max _{k \leq n}\left|Y_{k}\right| \geq \lambda\right\}$. The next result gives a bound on $\operatorname{Pr}\left\{L_{n} \geq \lambda\right\}$.

Lemma 3. ([3, p. 106].) Let $m_{i j k}$ and $L_{n}$ be as given in Lemma 2. Suppose that $\alpha>\frac{1}{2}$ and $\beta \geq 0$ and that $u_{1}, \ldots, u_{n}$ are nonnegative numbers such that

$$
\operatorname{Pr}\left\{m_{i j k} \geq \lambda\right\} \leq \frac{1}{\lambda^{4 \beta}}\left(\sum_{i<l \leq k} u_{l}\right)^{2 \alpha}, \quad 0 \leq i \leq j \leq k \leq n,
$$

for $\lambda>0$. Then

$$
\operatorname{Pr}\left\{L_{n} \geq \lambda\right\} \leq \frac{K}{\lambda^{4 \beta}}\left(\sum_{0<l \leq n} u_{l}\right)^{2 \alpha}
$$

for $\lambda>0$, where the constant $K \equiv K_{\alpha, \beta}$ depends only on $\alpha$ and $\beta$. 
Now we return to the process $S_{n}=\sum_{k=1}^{n} H_{k} G_{k}$, defined in Section 3. We want to verify that the sequence $Y_{n}=H_{n} G_{n}$ satisfies the conditions imposed on $m_{i j k}$ in Lemma 3, in particular for $\alpha=1$ and $\beta=1$. For $0<i<j<k<n$ and $\lambda>0$, we have

$$
\begin{aligned}
\operatorname{Pr}\left\{m_{i j k} \geq \lambda\right\}= & \operatorname{Pr}\left\{\left|S_{j}-S_{i}\right| \geq \lambda,\left|S_{k}-S_{j}\right| \geq \lambda\right\} \\
= & \operatorname{Pr}\left\{\left|S_{j}-S_{i}\right| \geq \lambda,\left|S_{k}-S_{j}\right| \geq \lambda, H_{j}=1\right\} \\
& +\operatorname{Pr}\left\{\left|S_{j}-S_{i}\right| \geq \lambda,\left|S_{k}-S_{j}\right| \geq \lambda, H_{j}=-1\right\} \\
= & 2 \operatorname{Pr}\left\{\left|S_{j}-S_{i}\right| \geq \lambda,\left|S_{k}-S_{j}\right| \geq \lambda, H_{j}=1\right\} \\
= & 2 \operatorname{Pr}\left\{\left|S_{j}-S_{i}\right| \geq \lambda,\left|S_{k}-S_{j}\right| \geq \lambda \mid H_{j}=1\right\} \operatorname{Pr}\left\{H_{j}=1\right\} \\
= & \operatorname{Pr}\left\{\left|S_{j-1}-S_{i}+G_{j}\right| \geq \lambda,\left|S_{k}-S_{j}\right| \geq \lambda \mid H_{j}=1\right\} \\
= & \operatorname{Pr}\left\{\left|S_{j-1}-S_{i}+G_{j}\right| \geq \lambda \mid H_{j}=1\right\} \operatorname{Pr}\left\{\left|S_{k}-S_{j}\right| \geq \lambda \mid H_{j}=1\right\} \\
= & \frac{\operatorname{Pr}\left\{\left|S_{j-1}-S_{i}+G_{j}\right| \geq \lambda, H_{j}=1\right\} \operatorname{Pr}\left\{\left|S_{k}-S_{j}\right| \geq \lambda, H_{j}=1\right\}}{\operatorname{Pr}\left\{H_{j}=1\right\}} \\
\leq & 4 \operatorname{Pr}\left\{\left|S_{j-1}-S_{i}+G_{j}\right| \geq \lambda\right\} \operatorname{Pr}\left\{\left|S_{k}-S_{j}\right| \geq \lambda\right\} \\
\leq & 4 \frac{\mathrm{E}\left(\left|S_{j-1}-S_{i}+G_{j}\right|^{2}\right)}{\lambda^{2}} \frac{\mathrm{E}\left(\left|S_{k}-S_{j}\right|^{2}\right)}{\lambda^{2}} .
\end{aligned}
$$

Note that the equality in (12) follows from the Markovian property of $H_{n}$, which implies that the past and the future, conditional on the present, are independent. Also, we have also used Chebyshev's inequality to obtain (13), which trivially holds if $i=j$ or $j=k$, because $m_{i j k}=0$ in either case. For the numerators on the right-hand side of (13), we have already computed $\mathrm{E}\left(S_{n}^{2}\right)=\operatorname{var}\left(S_{n}\right)=\operatorname{var}\left(\sum_{l=1}^{n} H_{l} G_{l}\right)$, in (9). A further simplification yields

$$
\begin{aligned}
\operatorname{var}\left(\sum_{l=1}^{n} H_{l} G_{l}\right) & =n A(\phi, w, v)^{2}+\frac{2 v^{2}}{(2 w-v)^{2}} \frac{(2 \phi-1)\left[(2 \phi-1)^{n}-1\right]}{(2 \phi-2)^{2}} \\
& \leq n A(\phi, w, v)^{2},
\end{aligned}
$$

where the inequality is due to the fact that $0 \leq 2 \phi-1<1$, and the quantity $A(\phi, w, v)^{2}$ is given in (10). Note that $A(\phi, w, v)^{2}$ is composed of $\mathrm{E}\left(G_{1}^{2}\right)$ and the nonnegative quantity $v^{2}(2 \phi-1) /(2 w-v)^{2}(1-\phi)$; thus, $\mathrm{E}\left(G_{1}^{2}\right) \leq A(\phi, w, v)^{2}$. Theorem 1 further implies that

$$
\begin{aligned}
\mathrm{E}\left(\left|S_{j-1}-S_{i}+G_{j}\right|^{2}\right) & =\mathrm{E}\left(\left(S_{j-1}-S_{i}\right)^{2}\right)+2 \mathrm{E}\left(\left(S_{j-1}-S_{i}\right) G_{j}\right)+\mathrm{E}\left(G_{j}^{2}\right) \\
& \leq(j-i-1) A(\phi, w, v)^{2}+\mathrm{E}\left(G_{j}^{2}\right) \\
& \leq(j-i) A(\phi, w, v)^{2} .
\end{aligned}
$$

Thus, continuing from (13) using the inequalities in (14) and (15), for $0 \leq i \leq j \leq k \leq n$ and $\lambda>0$ we have

$$
\begin{aligned}
\operatorname{Pr}\left\{m_{i j k} \geq \lambda\right\} & \leq 4 \frac{(j-i) A(\phi, w, v)^{2}}{\lambda^{2}} \frac{(k-j) A(\phi, w, v)^{2}}{\lambda^{2}} \\
& \leq 4 \frac{(k-i)^{2} A(\phi, w, v)^{4}}{\lambda^{4}} \\
& =\frac{1}{\lambda^{4}}\left[\sum_{i<l \leq k} 2 A(\phi, w, v)^{2}\right]^{2}
\end{aligned}
$$


The second inequality in (16) shows that $Y_{n}=H_{n} G_{n}$ satisfies the assumptions imposed on $m_{i j k}$ in Lemma 3, with $\alpha=1, \beta=1$, and $u_{l}=2 A(\phi, w, v)^{2}$. Therefore, it follows from that lemma that

$$
\operatorname{Pr}\left\{L_{n} \geq \lambda\right\} \leq \frac{K}{\lambda^{4}}\left[\sum_{0<l \leq n} 2 A(\phi, w, v)^{2}\right]^{2}=\frac{4 K A(\phi, w, v)^{4}}{\lambda^{4}} n^{2}
$$

for $\lambda>0$ and some fixed constant, $K$.

To obtain an upper bound on $\operatorname{Pr}\left\{\max _{k \leq n}\left|H_{k} G_{k}\right| \geq \lambda\right\}$, we use the strict stationarity of $H_{n} G_{n}$ to obtain

$$
\operatorname{Pr}\left\{\max _{k \leq n}\left|H_{k} G_{k}\right| \geq \lambda\right\} \leq n \operatorname{Pr}\left\{\left|H_{1} G_{1}\right| \geq \lambda\right\}=n \operatorname{Pr}\left\{\left|G_{1}\right| \geq \lambda\right\} \leq \frac{\mathrm{E}\left(G_{1}^{2}\right)}{\lambda^{2}} n .
$$

Applying Lemma 2 together with (17) and (18) yields

$$
\begin{aligned}
& \operatorname{Pr}\left\{\max _{|k-\lfloor n \theta\rfloor| \leq \varepsilon^{3}\lfloor n \theta\rfloor}\left|S_{k}-S_{\lfloor n \theta\rfloor}\right| \geq \varepsilon \sqrt{\lfloor n \theta\rfloor}\right\} \\
& \quad \leq \operatorname{Pr}\left\{L_{2 \varepsilon^{3}\lfloor n \theta\rfloor} \geq \frac{\varepsilon}{4} \sqrt{\lfloor n \theta\rfloor}\right\}+\operatorname{Pr}\left\{\max _{k \leq 2 \varepsilon^{3}\lfloor n \theta\rfloor}\left|H_{k} G_{k}\right| \geq \frac{\varepsilon}{4} \sqrt{\lfloor n \theta\rfloor}\right\} \\
& \quad \leq 4 K A(\phi, w, v)^{4} \frac{\left(2 \varepsilon^{3}\lfloor n \theta\rfloor\right)^{2}}{((\varepsilon / 4) / \sqrt{\lfloor n \theta\rfloor})^{4}}+\mathrm{E}\left(G_{1}^{2}\right) \frac{2 \varepsilon^{3}\lfloor n \theta\rfloor}{((\varepsilon / 4) / \sqrt{\lfloor n \theta\rfloor})^{2}} \\
& \quad=4096 K A(\phi, w, v)^{4} \varepsilon^{2}+32 \mathrm{E}\left(G_{1}^{2}\right) \varepsilon .
\end{aligned}
$$

Because $\varepsilon$ is arbitrary, (11) implies that

$$
\frac{S_{N_{n}}-S_{\lfloor n \theta\rfloor}}{\sqrt{\lfloor n \theta\rfloor}} \rightarrow 0 \text { in probability as } n \rightarrow \infty .
$$

This completes the demonstration of the central limit property for $S_{N_{n}}$; we state this main result below.

Theorem 4. Under the MEUD, the net difference process $\Delta_{n}$, defined in (8), has the central limit property that

$$
\mathcal{L}\left(\frac{\Delta_{n}}{A \sqrt{n \theta}}\right) \rightarrow \mathrm{N}(0,1),
$$

where $A=A(\phi, w, v)$ is as given in Theorem 2 and $\theta$ is given in Theorem 3 .

\section{Concluding remarks}

Under the modified Ehrenfest urn design that prescribes there to be a specific integer minimum number of balls in each urn, the process, $\Delta_{n}$, recording the treatment assignment differences has a dramatically different asymptotic balance property than that under the ordinary Ehrenfest urn design. In this sense of weak convergence, the balance property of $\operatorname{MEUD}(w, v)$ is not very different from the repeated simple random sampling design, except that the normalized difference process $\Delta_{n} / \sqrt{n}$ has a smaller asymptotic variance under the former than it does under the latter. For example, with $w=5$ and $v=1$ the asymptotic variance of $\Delta_{n} / \sqrt{n}$ under $\operatorname{MEUD}(w, v)$ is about $0.046765^{2}$. We have used a central limit theorem for dependent random variables, renewal theory, and two Kolmogorov-type maximal inequalities to establish 
the central limit property of $\Delta_{n}$ under $\operatorname{MEUD}(w, v)$. A project for future research is to apply the results obtained here to compare the balance and randomness properties of the MEUD with other sampling schemes.

We would also like to raise a question about the limiting process. As pointed out in [14, pp. 170-173], the EUD will yield the Ornstein-Uhlenbeck process when the time between ball transitions becomes small and the number of balls becomes large (also see [13] and [17]). An avenue of future research is to look at the resulting process when $\operatorname{MEUD}(w, v)$ undergoes the same limit operation.

Finally, we would like to remark on how the modification discussed here changes the underlying probabilistic structure of the EUD. It is well known that we can represent the EUD by a simple random walk on a finite Abelian group [7, pp. 19-20]. This group representation provides an elegant way of diagonalizing the transition matrix and finding the high-order transition probabilities in closed form for the Ehrenfest chain. Some variants of the EUD preserve this group structure [19], [10, pp. 52-53]. However, this group structure does not hold under $\operatorname{MEUD}(w, v)$. Another possibility for future research is to diagonalize the transition matrix of the Markov chain (either $W_{n, 1}$ or $\left.W_{n, 2}\right)$ under $\operatorname{MEUD}(w, v)$.

\section{References}

[1] Billingsley, P. (1968). Convergence of Probability Measures. John Wiley, New York.

[2] Billingsley, P. (1995). Probability and Measure, 3rd edn. John Wiley, New York.

[3] Billingsley, P. (1999). Convergence of Probability Measures, 2nd edn. John Wiley, New York.

[4] Bingham, N. H. (1991). Fluctuation theory for the Ehrenfest urn. Adv. Appl. Prob. 23, 598-611.

[5] Blackwell, D. and Hodges, J. L., JR. (1957). Design for the control of selection bias. Ann. Math. Statist. 28, 449-460.

[6] Chen, Y.-P. (2000). Which design is better? Ehrenfest urn versus biased coin. Adv. App. Prob. 32, 738-749.

[7] Diaconis, P. (1988). Group Representations in Probability and Statistics. Institute of Mathematical Statistics, Hayward, CA.

[8] Ehrenfest, P. and Ehrenfest, T. (1907). Über zwei bekannte Einwände gegen das Boltzmannsche H-Theorem. Phys. Z. 8, 311-314.

[9] Feller, W. (1968). An Introduction to Probability Theory and Its Applications, Vol. 1, 3rd edn. John Wiley, New York.

[10] Hoel, P. G., Port, S. C. And Stone, C. J. (1972). Introduction to Stochastic Processes. Houghton Mifflin, Boston, MA.

[11] Ibragimov, I. (1962). Some limit theorems for stationary processes. Theor. Prob. Appl. 7, 349-382.

[12] Ibragimov, I. (1963). A central limit theorem for a class of dependent random variables. Theor. Prob. Appl. 8, 83-89.

[13] Karlin, S. And McGregor, J. (1959). Random walks. Illinois J. Math. 3, 66-81.

[14] Karlin, S. and Taylor, M. H. (1981). A Second Course in Stochastic Processes. Academic Press, New York.

[15] Palacios, J. L. (1993). Fluctuation theory for the Ehrenfest urn via electric networks. Adv. Appl. Prob. 25, 472-476.

[16] Palacios, J. L. (1994). Another look at the Ehrenfest urn via electric networks. Adv. Appl. Prob. 26, 820-824.

[17] Sснасн, S. (1971). Weak convergence results for a class of multivariate Markov processes. Ann. Math. Statist. 42, 451-465.

[18] Serfling, R. J. (1968). Contributions to central limit theory for dependent variables. Ann. Math. Statist. 39, 1158-1175.

[19] Van Beek, K. W. H. and Stam, A. J. (1987). A variant of the Ehrenfest model. Adv. App. Prob. 19, $995-996$. 